\title{
Nutrition and Life Style of Selected Mild Hypercholesterolemic Patients
}

\author{
K. Anooja Thomas ${ }^{1 *}$ and P. Lincy ${ }^{2}$ \\ 'Associate Professor, Department of Home Science, CMS College, Kottayam, India; anoojam@gmail.com, \\ 2Department of Home Science, CMS College, Kottayam, India; lincyklmp@gmail.com
}

\begin{abstract}
A community based study in Kerala funded by World Health Organization reported hypercholesterolemia in 54.1 percent.The present study aimed to investigate the nutritional profile and life style behaviour of selected mild hypercholesterolemia adults.Two hundred adults irrespective of sex were randomly selected from Kottayam district kerala.Information on economic background,anthropometric mesurements, diet history, life style habit and medical history of the subjects were collected using an interview schedule. All the subjects were screened for total cholestrol to select mild hypercholestrolemic patients. Data collected were consoildated and tabulated. Among the two hundred subjects 20 subjectas were found to be mild hypercholestrolemic. All the selected subjects were doing moderate workonly. Twelve of the selected subjects were overweight followed by eight normal. Moderate working habit, lack of physical activity and faulty food habit were found to be the main reason for hypercholestrolemic. Supplementation and nutrition education are the strategies to correct mild hypercholestrolemic.
\end{abstract}

Keywords: Adults, Hypercholesterolemia, Mild Hypercholesterolemic

\section{Introduction}

Hypercholesterolemia refers to elevated levels of total cholesterol in the blood. Epidemiological investigations of human populations incriminate high levels of LDL cholesterol as being atherogenic [1]. Hypercholesterolemia is treated by reducing dietary cholesterol intake, administration of certain medications and rarely with other treatments including surgery. Foods high in saturated fats and cholesterol is the most common cause of hypercholesterolemia as excess of saturated fats may modestly increase LDL cholesterol level. Lack of physical activities also leads to increase in fat and cholesterol level. Diet plays an important role in offering health beneficial properties with increased physiological significance beyond the pure nutritional requirement. Dietary components presents specific bioactive components which indeed constitutes the potential ingredients of functional or health promoting foods. From time immemorial, man depended on plants as a source of food and also for all his medicinal needs. The present study was undertaken to (i) find out mild Hypercholesterolemic patients in Kottayam district, Kerala (ii) study the nutritional status and lifestyle behavior of mild Hypercholesterolemic patients.

\section{Methodology}

Two hundred adults irrespective of sex from Puthupalli and Ettumanur area of Kottayam district Kerala was selected for the study. Socio economic details, anthropo metric measurements, lifestyle behavior, dietary pattern and medical history of the subjects were collected using an interview schedule All the selected subjects were screened for total cholesterol. Among them mild hyper cholesterolemic patients were selected for further study.

${ }^{*}$ Author for correspondence 


\section{Results}

\subsection{Socio Economic Background of the Selected Subjects}

The Socio economic background of the selected subjects was studied with reference to their age, sex, educational status and .monthly income. The socio-economic background of the selected subjects is given in Table 1 .

Table 1. Socio-economic background of the selected subject

$\mathrm{N}=200$

\begin{tabular}{|c|c|c|}
\hline Particulars & $\begin{array}{c}\text { Number of } \\
\text { Subjects }\end{array}$ & Percent \\
\hline Age in years & 31 & 15 \\
$15-30$ & 99 & 50 \\
$31-45$ & 70 & 35 \\
$46-60$ & 40 & 20 \\
Sex & 160 & 80 \\
Male & 153 & 76 \\
Female & 40 & 20 \\
Educational Status & 7 & 4 \\
High School & & \\
Under Graduate & & 3 \\
Post Graduate & 6 & 50 \\
\hline${ }^{*}$ Monthly Income & 100 & 42 \\
Economicallyweaker & 85 & 5 \\
section & 9 & \\
Low income group & & \\
Middle income & & \\
group & & \\
High income group & & \\
${ }^{*}$ HUDCo [2]
\end{tabular}

Majority of the subjects belonged to 31-45 years followed by 46-60 years. A study conducted by Sawant [3] says that the increase of prevalence of hypercholesterolemia was more prominent in 31-40 age groups than in below 30 age groups. None of the subjects were illiterate and 153 subjects completed high school education. According to HUDCO [2] classification of monthly income majority were in low income group with monthly income between 3301-7300 rupees. Occupational status of the screened subjects (20) was

analyzed to know their activity level and it was found that all the screened subjects were moderate working only.

\subsection{Meal Pattern of the Selected Subject}

Figure 1 represents the percentage distribution of meal pattern of the selected subjects and it is clear that 94 percent of the selected subjects were non-vegetarians and majority had 3 main meals per day. Four percent of them were lacto-vegetarians who consume milk.

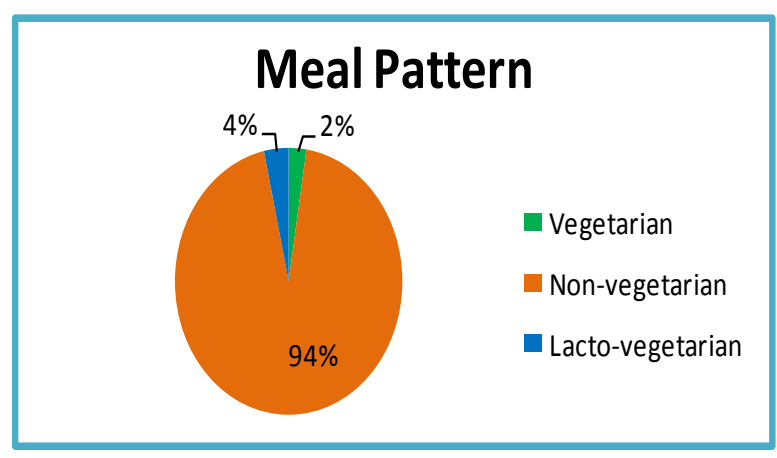

Figure 1. Meal pattern of the selected subjects 3.3 Selection of Mild Hypercholesterolemic Patients

\subsubsection{Total Cholesterol of the Selected Subjects}

Table 2. Categorization of selected subjects according to total cholesterol level

\begin{tabular}{|c|c|c|c|}
\hline $\begin{array}{c}* \\
* \\
\text { Lholesterol } \\
\text { Level }\end{array}$ & Category & Number & Percent \\
\hline$<200 \mathrm{mg} / \mathrm{dl}$ & Desirable & 163 & 82 \\
\hline $\begin{array}{c}200-239 \mathrm{mg} / \\
\mathrm{dl}\end{array}$ & Border line & 20 & 10 \\
\hline$\geq 240 \mathrm{mg} / \mathrm{dl}$ & High & 17 & 8 \\
\hline
\end{tabular}

${ }^{*}$ AHA [4]

Table 2 represents categorization of selected subjects according to total cholesterol level andit is clear that 20 subjects were mild hypercholesterolemic when classified as per American Heart Association [4].

\subsubsection{Lifestyle Behavior}

Life style behavior of the screened subjects were studied through their habits of doing exercise, alcohol consumption, smoking pattern and eating away from home.

\subsubsection{Exercise Pattern of Screened Subjects}

While analyzing the exercise pattern of screened subjects it was found that eight of them were going for walking occasionally followed by four subjects rarely. Others were doing household activities only 
A study conducted by NCEP says that increased physical activity has beneficial effects on several metabolic risk factors including insulin resistance, blood pressure and serum triglycerides, LDL, and HDL levels [5]..

\subsubsection{Alcohol Consumption and Smoking Pattern}

All the screened subjects were women and they didn't have any habit of alcohol consumption and smoking.

\subsubsection{Eating Away from Home}

It was found that nine of the screened subjects were eating away from home occasionally and they were preferring snack items.

\subsubsection{Dietary Habits of the Screened}

\section{Subjects}

Dietary habits were assessed through meal pattern, likes and dislikes, food frequency and 24 hour recall

\subsubsection{Meal Pattern of Screened Subjects}

Figure 2 brings out the meal pattern of screened subjects.

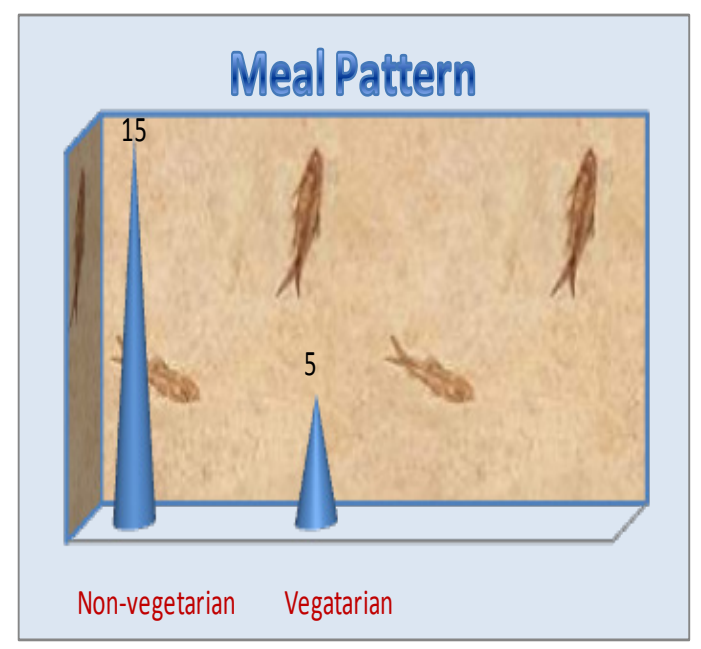

Figure 2. Meal pattern of screened subjects.

Among the twenty subjects 15 were non-vegetarians taking fish daily and other meat products in weekly or monthly basis. All of them were taking 3 meal pattern and they were consuming tea with some snacks in the evening.

\subsubsection{Fatty Food Consumption of Screened Subjects}

The main source of saturated fat found to be was coconut and its products. The non-vegetarian sub- jects consumed egg with out removing the yolk which is the rich source of cholesterol (Table 3)

Table 3. Fatty food consumption of screened subjects

\begin{tabular}{|l|c|c|c|c|}
\hline Food stuffs & Daily & Frequently & Occasionally & Rarely \\
\hline Coconut oil & 11 & 2 & 4 & 3 \\
\hline Palm oil & 2 & 3 & - & - \\
\hline Vegetable oil & 1 & 2 & - & - \\
\hline Coconut & 16 & 4 & - & - \\
\hline Milk (whole) & 14 & 3 & 2 & 1 \\
\hline Curd & 5 & 4 & & 2 \\
\hline Butter & - & - & 5 & 6 \\
\hline Beef & - & 1 & 10 & 3 \\
\hline Chicken & - & 6 & 7 & 2 \\
\hline Egg (whole) & & 12 & 2 & 1 \\
\hline
\end{tabular}

A study conducted by Mensink et al. [6] found thatMonounsaturated or polyunsaturated fat lowers LDL cholesterol.

\subsubsection{Mean Nutrient Intake}

Mean nutrient intake of screened subjects were calculated and compared with Recommended Dietary Allowances of Moderate working women [7] and it is presented in Figure 3. It was observed that mean intake of iron, calcium and riboflavin were below Recommended Dietary Allowances and the intake of fat is 64 percent higher than RDA [7].

\subsubsection{Anthropometric Measurements}

Height, weight, waist and hip of the screened subjects were measured and calculated Body Mass Index and Waist Hip Ratio.

\subsubsection{Categorization of Screened Subjects Based on BMI}

Figure 4 represents the categorization of screened subjects according to Body Mass Index classification of WHO [8].

From the Figure 4 it is clear that among the twenty subjects 12 were overweight followed by 8 normal. A study conducted by Johnson RK et al. [9] says that much of the increase in serum triglycerides that occurs in adult life is caused by weight gain, lack of exercise, and a diet rich in simple carbohydrates and sugar sweetened beverages.

From Table 4 it is clear that five of the screened subjects were suffering from hyper tension followed three were suffering from diabetes. Both were didn't take any medication for the same. 


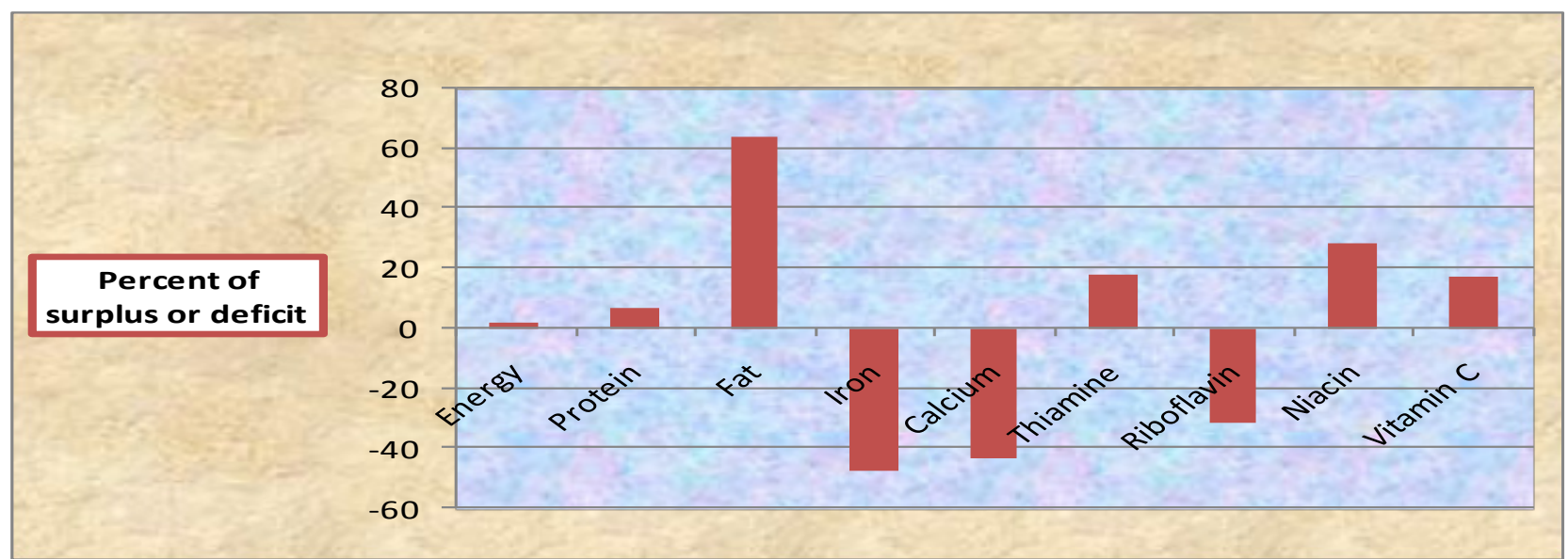

Figure 3. Mean nutrient intake of screened subjects.

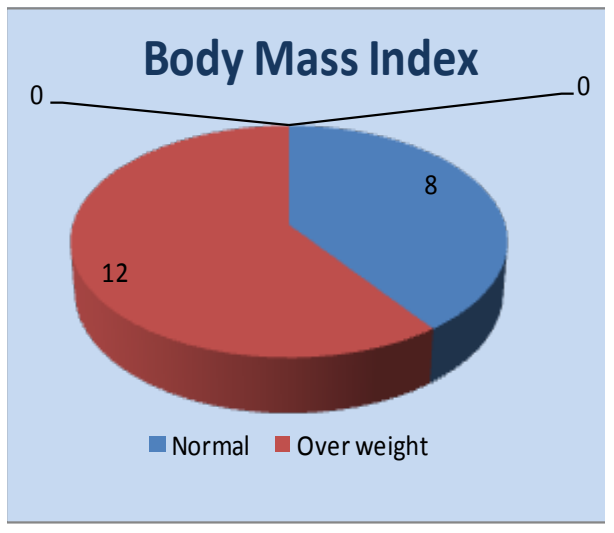

Figure 4. Distribution of screened subjects according to BMI.

\subsubsection{Medical History of the Screened}

\section{Subjects}

Table 4. Medical history of screened subjects

\begin{tabular}{|c|c|}
\hline Disease condition & No. of subjects \\
\hline Diabetes & 3 \\
\hline Hypertension & 5 \\
\hline Kidney disease & 2 \\
\hline Hyper thyroids & 2 \\
\hline No illness & 8 \\
\hline
\end{tabular}

\section{Conclusion}

Moderate working habit, lack of physical activity and faulty food habit were found to be the main reason for hypercholesterolemia. Supplementation and nutrition education are the strategies to correct mild hypercholesterolemia.

\section{References}

1. Third report of the National Cholesterol Education Programme, "Evaluation and treatment of high blood cholesterol in adults", NIH Publication; p. II-2, 2002.

2. "HUDCO monthly income classification", 2007.

3. Sawant A. M., Shetty D., Mankeshwar R., and Ashavaid T. F., "Prevalence of dyslipidemia in young adult Indian population", J Assoc Physicians India . vol. 56, p. 99-102, 2008.

4. American Heart Association, 2010.Availaible: http:// www.heart.org/cholesterol

5. Third Report of the National Cholesterol Education Program (NCEP) Expert Panel on Detection, "Evaluation, and Treatment of High Blood Cholesterol in Adults (Adult Treatment Panel III), Final report", Circulation, vol. 106(25), p. 3143-3421, 2002.

6. Mensink R. P., Zock P. L., Kester A. D. M., and Katan M. B., "Effects of dietary fatty acids and carbohydrates on the ratio of serum total to HDL cholesterol and on serum lipids and apolipoproteins", Am. J. Clin. Nutr., vol. 77(5), p. 1146-1155, 2003.

7. "Recommended dietary allowances for Indians", ICMR, 2010.

8. World Health Organization, "Classification of Body Mass Index", 2009.

9. Johnson RK, Appel LJ, Brands M,Howard BV,Lefever M,Lustig RH, Sacks F, Steffen LM, and Wylie-Rosett J. Dietary sugars intake and cardiovascular health: a scientific statement from the America Heart Association, 2009, Circulation.,vol.120(11), p.10111020 